\title{
Reviewer Acknowledgment
}

The Journal of Theoretical and Philosophical Psychology depends on the goodwill of many experts who help review manuscripts that have been submitted for publication. In addition to the Consulting Editors, the following individuals reviewed manuscripts that were considered from September 2015 to August 2016. The Editor acknowledges all reviewers and thanks them for their time and effort.

Mark H. Bickhard

Simon Boag

John Christopher

Evangelia Chrysikou

Courtenay Crouch

Lucian Delescu

Alvin C. Dueck

Rich Duus

Sayyed Mohsen Fatemi

Susi Ferrarello

Lisa Danner Finlay

Leopoldina Fortunati

Blaine Fowers

Mark Freeman

Edwin E. Gantt

Kenneth Gergen

Gary Gregg

George Guim

Gregg Henriques

Jack Huntington
Kwang-Kuo Hwang

Bruce Jennings

Paul Kiritsis

James T. Lamiell

Tyler Lefevor

Rachel Liebert

Ram Mahalingam

Kareen Malone*

Peter Marshall

Monica Martin

Dan McAdams

Judith Mendell

Megan Meyer

Ronald B. Miller

Rune Moelbak

Matthew Mosconi

Joseph Ostenson*

Tim Racine

Christopher H. Ramey
Jeffrey Reber*

Frank C. Richardson

Daniel Robinson

Armin Schultz

Albert Silverstein

Laurence Smith

Anna Stetsenko

Brett Stoudt

Donna Tafreshi

Thomas Teo

René Van Hezewijk

Mostafa Vaziri

Fred Weizmann

Dennis C. Wendt

Fred Wertz

Brady Wiggins

Stephen Yanchar

Milica Zegarac

* Denotes a reviewer who coreviewed under the supervision of a primary reviewer. 